\title{
Erratum: LISA for cosmologists: Calculating the signal-to-noise ratio for stochastic and deterministic sources [Phys. Rev. D 100, 104055 (2019)]
}

\author{
Tristan L. Smith॰ and Robert R. Caldwell
}

(Received 28 September 2021; published 21 January 2022)

DOI: 10.1103/PhysRevD.105.029902

In preparation for our paper, a factor of $1 / 2$ was inconsistently implemented in the definition of the quantity $F_{A_{B C}}^{P}$ in Eq. (11). This does not change the validity of the derivations that we present, but does impact the examples in which we compute the signal to noise ratio (SNR). The factor of $1 / 2$ means that Eq. (14) gives the detector response per one-way interferometer path, whereas Eqs. (55) and (56) give the detector noise per round-trip path. A simple way to make the detector noise consistent with the response functions is to reduce the noise spectral densities in Eqs. (53) and (54) by a factor of 4 , since both expressions are quadratic in path length:

$$
\begin{gathered}
S_{I} \rightarrow S_{I} / 4 \\
S_{I I} \rightarrow S_{I I} / 4 .
\end{gathered}
$$

With this change the revised Eqs. (55) and (56) give the detector noise per one-way path. This change brings the numerical values of the SNR into agreement with other computations in the literature (e.g., Refs. [1-3]).

In Sec. VIIIA for the example of a flat stochastic gravitational wave background (SGWB), we obtain $\Omega_{\mathrm{GW}}=1.18 \times 10^{-13}$ or $\Omega_{\mathrm{GW}} h^{2}=5.30 \times 10^{-14}$ for $T=4 \mathrm{yr}$, and $\Omega_{\mathrm{GW}}=1.36 \times 10^{-13}$ or $\Omega_{\mathrm{GW}} h^{2}=6.12 \times 10^{-14}$ for $T=3 \mathrm{yr}$.

In Sec. VIIIC, for the example of binary inspiral in a GW150914-like system using Eqs. (86) and (87) the SNR is 4.64, 6.72 for 4, $10 \mathrm{yr}$, whereas using Eqs. (85) and (86) the SNR is 5.33,7.51.

In Sec. VIIID, for the example of a monotone binary, the noise power spectral density is $\Sigma_{h}\left(f_{m}\right)=7.2 \times 10^{-41} \mathrm{~Hz}^{-1}$. For $T=1,4$ yr observation, the sky- and polarization-averaged SNR is 80, 160 .

We list all of the quantities that are affected by this change in Table I. The minimum detectable amplitudes all refer to $\mathrm{SNR}=5$.

A fully revised manuscript may be found on the arXiv or obtained through a request to the authors.

TABLE I. List of all quantities that are affected by the corrected response function.

\begin{tabular}{lcc}
\hline \hline Corrected quantitative results & \\
\hline Quantity & Value quoted in original paper & Corrected value \\
\hline Minimum detectable SGWB amplitude $100 \%$ duty cycle $\left(\Omega_{\mathrm{GW}}\right)$ & $4.7 \times 10^{-13}$ & $1.18 \times 10^{-13}$ \\
Minimum detectable SGWB amplitude $100 \%$ duty cycle $\left(\Omega_{\mathrm{GW}} h^{2}\right)$ & $2.1 \times 10^{-13}$ & $5.30 \times 10^{-14}$ \\
Minimum detectable SGWB amplitude $75 \%$ duty cycle $\left(\Omega_{\mathrm{GW}}\right)$ & $5.4 \times 10^{-13}$ & $1.36 \times 10^{-13}$ \\
Minimum detectable SGWB amplitude $75 \%$ duty cycle $\left(\Omega_{\mathrm{GW}} h^{2}\right)$ & $2.4 \times 10^{-13}$ & $6.12 \times 10^{-14}$ \\
Minimum detectable integrated SGWB amplitude four-year mission $\left(\Omega_{\mathrm{GW}}\right)$ & $2.7 \times 10^{-13}$ & $1.2 \times 10^{-13}$ \\
SNR for GW150914 $T=4 \mathrm{yr}(10 \mathrm{yr})$ before merger, Eqs. $(86),(87)$ & $2.7(3.8)$ & $4.64(6.72)$ \\
SNR for GW150914 $T=4 \mathrm{yr}(10 \mathrm{yr})$ before merger, Eqs. $(85),(86)$ & $1.5 \times 10^{-40} \mathrm{~Hz}^{-1}$ & $7.33(7.51)$ \\
Noise power spectral density $\left[\Sigma_{h}\left(f_{m}\right)\right]$ for ZTFJ1539 & $40(80)$ & $80(160)$ \\
SNR for ZTFJ1539 $T=1 \mathrm{yr}(4 \mathrm{yr})$ & \\
\hline \hline
\end{tabular}


[1] A. Sesana, Prospects for Multiband Gravitational-Wave Astronomy after GW150914, Phys. Rev. Lett. 116, 231102 (2016).

[2] T. Robson, N. J. Cornish, and C. Liu, The construction and use of LISA sensitivity curves, Classical Quantum Gravity 36, 105011 (2019).

[3] T. B. Littenberg and N. J. Cornish, Prospects for gravitational wave measurement of ZTF J1539+ 5027, Astrophys. J. Lett. 881, L43 (2019). 